ИНСТИТУТ ПО МАТЕМАТИКА С ИЗЧИСЛИТЕЛЕН ЦЕНТЬР INSTITUTE OF MATHEMATICS WITH COMPUTER CENTER

\title{
Smooth functions in Orlicz function spaces
}

БЪЛГАРСКА АКАДЕМИЯ HA НАУКИТЕ

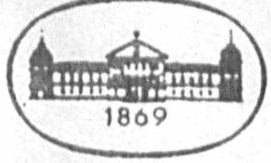

BULGARIAN ACADEMY OF SCIENCES
Raquel Gonzalo Rumen Maleev

\author{
Preprint \\ March 1996 \\ No 5
}

Department of Mathematical Modelling 


\title{
Smooth functions in Orlicz function spaces
}

\author{
R. Gonzalo R.P. Maleev*
}

\begin{abstract}
The best order of $C^{k}$-smoothness is found for the Orlicz spaces $L_{M}(0,1)$ and $L_{M}(0, \infty)$.

Mathematics Subject Classifications. Primary 46B20,46N25.

Key words. Orlicz spaces. Fréchet differentiability, smoothness.
\end{abstract}

\section{Introduction.}

The problem of the existence of smooth bump functions on a Banach is closely related to many problems in the nonlinear analysis, like existence of smooth partitions of the unity, approximations etc. Several deep results of Deville ([De1], [De2]) show the geometric impact of the existence of higher order Fréchet differentiable bumps is Banach spaces that do not contain isomorphic copies of $c_{0}$. As a Fréchet smooth norm immediately produces a bump of the same order of smoothness all results on smooth renorming give positive results for bumps. An extensive treatment on the existence of higher order Fréchet differentiable norms for the classical spaces can be found in [DGZ]. The converse problem, namely to find upper (sharp if possible) bound for the order of smoothness of bumps in a Banach space seems to be more difficult. In $[\mathrm{BF}]$ and $[\mathrm{MTr}]$ it is solved for $L_{p}$-spaces, $1<p<\infty$, and for Orlicz sequence spaces $h_{M}, 0<\alpha_{M}^{0} \leq \beta_{M}^{0} \leq \infty$, respectively.

Our aim is to investigate this problem for the Orlicz function spaces $L_{M}(0,1)$ and $L_{M}(0, \infty)$.

We begin with some notations and definitions. Troughout the paper $X, Y$ are Banach spaces and differentiability is understood in Fréchet sense.

*Research supported for the second author in part by the Bulgarian Ministry of Education, Science and Technology, Contract MM-409/94. 
The class of all k-times continuously differentiable functions $f: X \rightarrow Y$ is denoted by $C^{k}(X, Y)$.

Let $\omega:[0, \infty) \rightarrow[0, \infty)$ have the properties

i) $\omega(t)=o\left(t^{k}\right), k \geq 1$;

ii) $\omega_{1}(t)=\omega(t) / t^{k}$ is non-decreasing and $\omega_{1}(\lambda t) \leq \lambda \omega_{1}(t), \lambda \geq 1$.

Definition. The function $f: X \rightarrow Y$ is said to be $H^{\omega}$-smooth in $V \subset X$ if $f \in C^{k}(V)$ and for every $x \in V$ there exist positive constants $r$ and $A$ ( $r$ and $A$ may depend on $x$ ), such that

$$
\left\|f^{(k)}(y)-f^{(k)}(z)\right\|<A \omega_{1}(\|y-z\|)=A \frac{\omega(\|y-z\|)}{\|y-z\|^{k}}
$$

for any $y, z \in B(x ; r) \cap V$.

We note that norm in the left-hand side is the norm of the k-linear symmetric form $f^{(k)}(y)-f^{(k)}(z)$.

If in (1) the constants $r$ and $A$ do not depend on $x$ the function $f$ is called $U H^{\omega}$-smooth. We recall that a non-zero real-valued function with bounded support is called bump function or simply bump. If in $X$ there is a $C^{k}\left(H^{\omega}, U H^{\omega}\right)$-smooth bump we say that $X$ is $C^{k}\left(H^{\omega}, U H^{\omega}\right)$-smooth. If $\omega(t)=t^{p}$ we say that $X$ is $H^{p}\left(U H^{p}\right)$-smooth.

We recall also that $M$ is Orlicz function, provided $M$ is even, convex and $M(0)=0, M(t)>0$ for $t \neq 0$. The space of all classes $x$ of equivalent $\mu$-measurable functions over the measure space $(S, \Sigma, \mu)$ such that

$$
\widetilde{M}(x / \lambda)=\int_{S} M(x(s) / \lambda) d \mu(s)<\infty
$$

for some $\lambda$, normed by $\|x\|=\inf \{\lambda>0 ; \widetilde{M}(x / \lambda) \leq 1\}$ is called Orlicz space and is denoted by $L_{M}(S, \Sigma, \mu)$. Two Orlicz functions $M$ and $N$ are called equivalent $(M \sim N)$ at 0 (at $\infty$, at 0 and $\infty)$ if there exists constant $c>0$, such that

$$
c^{-1} M\left(c^{-1} t\right) \leq N(t) \leq c M(c t), t \in[0,1]([1, \infty),[0, \infty)) .
$$

In the following we deal with Orlicz sequence spaces $l_{M}$ and with Orlicz function spaces $L_{M}(0,1)$ and $L_{M}(0, \infty)$. The smoothness properties of 
these spaces essentially depend on their Boyd indices:

$$
\begin{aligned}
& \alpha_{M}^{0}=\sup \left\{p ; \sup \left\{u^{-p} M(u v) /(M(v) ; u, v \in(0,1]\}<\infty\right\},\right. \\
& \beta_{M}^{0}=\inf \left\{p ; \inf \left\{u^{-p} M(u v) /(M(v) ; u, v \in(0,1]\}>0\right\}\right.
\end{aligned}
$$

for $l_{M}$

$$
\begin{aligned}
& \alpha_{M}^{\infty}=\sup \left\{p ; \sup \left\{u^{p} M(v) / M(u v) ; u, v \geq 1\right\}<\infty\right\} \\
& \beta_{M}^{\infty}=\inf \left\{p ; \inf \left\{u^{p} M(v) / M(u v) ; u, v \geq 1\right\}>0\right\}
\end{aligned}
$$

for $L_{M}(0,1)$ and

$$
\alpha_{M}=\min \left(\alpha_{M}^{0}, \alpha_{M}^{\infty}\right), \quad \beta_{M}=\max \left(\beta_{M}^{0}, \beta_{M}^{\infty}\right) .
$$

for $L_{M}(0, \infty)$.

We note that always $\alpha_{M} \leq \beta_{M}$.

The function $M$ is said to satisfy the $\Delta_{2}$-condition at $0(\infty, 0$ and $\infty)$ if $\beta_{M}^{0}\left(\beta_{M}^{\infty}, \beta_{M}\right)<\infty$.

We consider only Orlicz spaces $l_{M}\left(L_{M}(0,1), L_{M}(0, \infty)\right)$, generated by Orlicz functions $M$ that satisfy the $\Delta_{2}$ - condition at 0 (at $\infty$, at 0 and $\infty)$.

The following results will be used in the sequel:

Proposition 1 ([BF]). Let $p \in(1, \infty), k=E(p)$ and $n$ be the usual norm in $L_{p}$. Then

i) $n \in C^{\infty}$, provided $p$ is even;

ii) $n \in U H^{p}$, provided $p$ is not even. In this case no bump in $L_{p}$ is $E(p)+1$ times differentiable, where

$$
E(p)= \begin{cases}{[p],} & p \text { is not integer } \\ p-1, & \text { otherwise. }\end{cases}
$$


Proposition 2 ([MT]). Let $\alpha_{M}^{0} \in(1, \infty)$ and $f$ be $k$-times differentiable bump in $l_{M}$, where $M \nsim t^{p}$ at 0 for some even $p$. Then

$$
k \leq E\left(\alpha_{M}^{0}\right) .
$$

Proposition 3 ([LT], [HR]). $l_{p}$ is isomorphic to a subspace

i) of $l_{M}$ iff $p \in\left[\alpha_{M}^{0}, \beta_{M}^{0}\right]$;

ii) of $L_{M}(0,1)$ iff $p \in\left[\alpha_{M}^{\infty}, \beta_{M}^{\infty}\right] \cup\left(\beta_{M}^{\infty}, 2\right) \cup\{2\}$,

iii) of $L_{M}(0,1)$ iff $p \in\left[\alpha_{M}^{0}, \beta_{M}^{0}\right] \cup\left[\alpha_{M}^{\infty}, \beta_{M}^{\infty}\right] \cup\left(\beta_{M}^{\infty}, \alpha_{M}^{0}\right) \cup\left(\beta_{M}^{\infty}, 2\right) \cup\{2\}^{1}$.

\section{Smooth bumps in $L_{M}(0,1)$ and $L_{M}(0, \infty)$.}

Our main result is the following

Theorem 1 Let $1<\alpha_{M}^{\infty} \leq \beta_{M}^{\infty}<\infty$ and $f \in C^{k}\left(L_{M}(0,1)\right)$, where at $\infty$ the function $M \nsim t^{2 n}, n \in \mathbb{N}$,. Then

$$
' k \leq E\left(\alpha_{M}^{\infty}\right) .
$$

Proof. Let us note first that, according to Proposition $3, l_{p}$ is isomorphic to a subspace of $L_{M}(0,1)$ for any $p \in\left[\alpha_{M}^{\infty}, \beta_{M}^{\infty}\right]$. If $\alpha_{M}^{\infty}$ is not an even integer, then (2) follows straightforward from Proposition 1. On the other hand, if $\alpha_{M}^{\infty}=2 n<\beta_{M}^{\infty}, n \in \mathbb{N}$, the Orlicz sequence space $l_{N}, N(t) \sim t^{2 n} /|\ln t|$ (see e.g. [LT1], Ex.4.c.2) is isomorphically embedded in $L_{M}(0,1)$ and (2) follows from Proposition 2.

The only nontrivial case is $\alpha_{M}^{\infty}=\beta_{M}^{\infty}=2 n, n \in \mathbb{N}$. Suppose $L_{M}(0,1)$ is $C^{2 n}$-smooth. Let $Y$ be arbitrary infinite dimensional subspace of $L_{M}(0,1)$. Let $\left\{x_{n}\right\}$ be a weakly null normalized sequence in $Y$, which does not contain norm convergent subsequence. This is always possible because $Y$ is reflexive.

Using an idea of Räbiger (see [R, p.82]) one can easily prove that $\left\{x_{n}\right\}$ has a subsequence $\left\{y_{n}\right\}$, that either has a lower 2-estimate or is equivalent to a disjoint sequence.

Assume first, that $\left\{y_{n}\right\}$ has a lower 2-estimate, i.e. there exists a constant $c>0$ such that

$$
c\left(\sum_{i=1}^{n}\left|a_{i}\right|^{2}\right)^{1 / 2} \leq\left\|\sum_{i=1}^{n} a_{i} y_{i}\right\|
$$

\footnotetext{
${ }^{1} \mathrm{An}$ interval $(\mathrm{a}, \mathrm{b})$, for $b \leq a$, means of course the empty set.
} 
for every $a_{1}, a_{2}, \ldots, a_{n} \in \mathbb{R}, n \in \mathbb{N}$. As $L_{M}(0,1)$ is $C^{2}$-smooth, according to [FWG], it is $U H^{2}$-smooth also, i.e. there is in $L_{M}(0,1)$ a $C^{1}$-smooth bump with uniformly Lipschitz derivative. Therefore, by using Proposition 1.1 in [GJ], we obtain that the sequence $\left\{y_{n}\right\}$ (passing to a subsequence if necessary) has also an upper 2-estimate:

$$
\left\|\sum_{i=1}^{n} a_{i} y_{i}\right\| \leq C\left(\sum_{i=1}^{n}\left|a_{i}\right|^{2}\right)^{1 / 2}
$$

for all $a_{1}, a_{2}, \ldots a_{n} \in \mathbb{R}, n \in \mathbb{N}$, where $C$ is a positive constant.

Obviously (3) and (4) imply $\left\{y_{n}\right\}$ is equivalent to the unit vector basis in $l_{2}$. Of course, $\left[\left\{y_{n}\right\}\right]$ is a subspace of $Y$ of cotype $2 n$.

Let us consider now the case $\left\{y_{n}\right\}$ is equivalent to a disjoint sequence $\left\{y_{n}^{\prime}\right\}$. Following Lindenstrauss and Tzafriri [LT, Prop.3] we find a subsequence of $\left\{y_{n}^{\prime}\right\}$ which is equivalent to the unit vector basis of $l_{G}$, where the Orlicz function $G$ is in

$$
C_{M}^{\infty}=\overline{c o n v}\left\{N \in C(0,1) ; N(t)=\lim _{n \rightarrow \infty} \frac{M\left(t_{n} t\right)}{M\left(t_{n}\right)} \text { for some }\left\{t_{n}\right\} \rightarrow \infty\right\} .
$$

The closure is norm closure in $C(0,1)$. It is not hard to check that $\alpha_{G}^{0}=\beta_{G}^{0}=2 n$. Now from [MT] immediately follows that $G \sim t^{2 n}$ at 0 , i.e. $\left\{y_{n}^{\prime}\right\}$ has a subsequence $\left\{z_{n}\right\}$ equivalent to the unit vector basis of $l_{2 n}$. As above $\left[\left\{z_{n}\right\}\right]$ is a subspace of $Y$ of cotype $2 n$.

What we prowed is that every infinite dimensional subspace $Y$ of $L_{M}(0,1)$ contains a subspace of cotype $2 n$, i.e. $L_{M}(0,1)$ is saturated with subspaces of cotype $2 n$. Our assumption that $L_{M}(0,1)$ is $C^{2 n}$-smooth, according to a result of Deville from [D1] (see e.g. [DGZ,T.V.5.1.]), leads to the conclusion that $L_{M}(0,1)$ is of cotype $2 n$ and has $C^{\infty}$-smooth bump. But $L_{M}(0,1)$ is supperreflexive and from [FDPZ, Cor.2] it follows that $L_{M}(0,1)$ has a separating polynomial.

Now from [GGJ] (there is proved that $L_{M}(0,1)$ admits a separating polynomial iff $M \sim t^{2 n}$ at $\infty$ for some $n \in \mathbb{N}$ ) it follows that $M \sim t^{2 n}$ at $\infty$, which is contradiction.

Thus Theorem 1 is proved.

Remark 1. The estimate (2) is sharp. Indeed, from ([M], Remark 4) it follows that in $L_{M}(0,1), 1<\alpha_{M}^{\infty} \leq \beta_{M}^{\infty}<\infty$, there exist an equivalent $H^{a}$-smooth norm for any $a \in\left(E\left(\alpha_{M}^{\infty}\right), \alpha_{M}^{\infty}\right)$ which is obviously $C^{k}$-smooth, $k=E\left(\alpha_{M}^{\infty}\right)$. 
Corollary 1 Let $1<\alpha_{M} \leq \beta_{M}<\infty$ and $f \in C^{k}\left(L_{M}(0, \infty)\right)$, where $M \not$ $\max \left(t^{2 m}, t^{2 n}\right), m, n \in \mathbb{N}$, at 0 and $\infty$. Then

i) $k \leq E\left(\alpha_{M}^{\infty}\right)$ if $\alpha_{M}$ even, $M \sim t^{\alpha_{M}}$ at 0 and $\alpha_{M} \leq E\left(\alpha_{M}^{\infty}\right)$,

ii) $k \leq E\left(\alpha_{M}\right)$ otherwise.

Proof. Let us observe first, that obviously the spaces $l_{M}$ and $L_{M}(0,1)$ are isometrically embedded in $L_{M}(0, \infty)$. Then i) follows directly from Theorem 1.

To prove ii) it is enough to use Propositions 1-3 and Theorem 1. Indeed, $L_{M}(0, \infty)$ contains an isomorphic copy of $l_{\alpha_{M}}$ and if $\alpha_{M}$ is not an even integer ii) follows from Proposition 1.

Suppose now $\alpha_{M}=2 n$.

The cases $\alpha_{M}=\alpha_{M}^{0}<\beta_{M}^{0}, \alpha_{M}=\alpha_{M}^{\infty}<\beta_{M}^{\infty}$ and $\alpha_{M}=\alpha_{M}^{\infty}=\beta_{M}^{\infty}<$ $\alpha_{M}^{0}$ can be treated similarily: as in the proof of Theorem 1 we have that $L_{M}(0, \infty)$ contains an isomorphic copy of $l_{N}, N \sim t^{\alpha_{M}} /|\ln t|$ and ii) follows from Proposition 2.

The last case to be considered is

$$
\alpha_{M}=\beta_{M}=2 m, m \in \mathbb{N} .
$$

From our assumptions it follows that either $M(t) \nsim t^{2 m}$ at 0 , or $M(t) \nsim t^{2 m}$ at $\infty$ and to obtain ii) it is enough to use one more time Proposition 1 or Theorem 1.

Corollary 1 is proved.

Remark 2. In the case $M \sim \max \left(t^{2 m}, t^{2 n}\right), m, n \in \mathbb{N}$ at 0 and $\infty$ we have that $L_{M}(0, \infty)$ is supperreflexive and isomorphic to the space $L_{2 m}(0, \infty) \cap L_{2 n}(0, \infty)$ equipped with the norm

$$
\left|\|x \mid\|=\max \left(\|x\|_{2 m},\|x\|_{2 n}\right),\right.
$$

as it is shown in $[\mathrm{H}]$.

It is easy to check that

$$
P(x)=\int_{0}^{\infty}(x(s))^{2 k} d \mu(s)+\int_{0}^{\infty}(x(s))^{2 n} d \mu(s)
$$

is a separating polynomial on $L_{M}(0, \infty)$ and therefore $L_{M}(0, \infty)$ is $C^{\infty}$ smooth. 
Remark 3. The estimate in ii) is sharp, which follows in the same way as in Remark 1 using ([M] Rem.4]). It turns out that the estimate in i) is also sharp as it follows from the following

Theorem 2 Let $M$ be Orlicz function equivalent at 0 to $t^{2 m}, m \in \mathbb{N}$ and $k=E\left(\alpha_{M}^{\infty}\right) \geq 2 m$. If $M \nsim t^{2 n}, m<n$ at $\infty$, then in $L_{M}(0, \infty)$ there exists an equivalent $H^{\omega}$-smooth norm, where

$$
\omega(t)=t^{k+1} \sup \left\{\frac{u^{k+1} M(v)}{M(u v)} ; u \in[1,1 / t], v \geq 1\right\}, t \in(0,1] .
$$

Proof. It is easy to check that the function

$$
N(t)=\int_{0}^{t} u^{-1} N_{1}(u) \exp \frac{u}{u-t} d u, N_{1}(t)=\int_{0}^{t} N_{0}(u) d u / u,
$$

where

$$
N_{0}(t)= \begin{cases}t^{2 n}, & t \in[0,1] \\ M(t) / M(1), & t \geq 1,\end{cases}
$$

is Orlicz function equivalent to $M$ at 0 and $\infty$. Moreover, $N$ is infinitely many times differentiable for $t \neq 0$ and for $i=1,2, \ldots$.

$$
t^{i}\left|N^{(i)}(t)\right| \leq c_{i} N\left(c_{i} t\right), \quad t \in \mathbb{R} \backslash\{0\},
$$

for some positive constants $c_{i}$.

Taking into account (5) and that

$$
N^{(i)}(t) \equiv 0, t \in[0,1]
$$

for $i>k$, we obtain the desired result repeating step by step the proof of Theorem 1 from $[\mathrm{M}]$.

Remark 4. We note that in $L_{M}(0, \infty), M \sim \max \left(t^{2 m}, t^{2 n}\right)$ there is even an equivalent $C^{\infty}$-smooth norm that improves the assertion from Remark 2 above. Indeed, $M$ is equivalent at 0 and $\infty$ to $N(t)=t^{2 m}+t^{2 n}$ and the statement is therefore easily obtained as above. 


\section{References}

[BF] Bonic, R. and J. Frampton.: Smooth functions on Banach manifolds, J. Math. Mech. 15 (1966), 877-898.

[De1] Deville, R.: Geometrical implications of the existence of very smooth bump functions in Banach spaces, Israel J. Math. 67 (1989), 1-22.

[De2] Deville, R.: A characterization of $C^{\infty}$-smooth Banach spaces, Bull. London Math. Soc. 22 (1990), 13-17.

[DeGZ] Deville, R., Godefroy, G. and V. Zizler: Smoothness and Renormings in Banach spaces, Pitman Monographs and Surveys in Pure and Applied Mathematics 64 (1993).

[FPWZ] Fabian, M., Preiss, D., Whitfield, J.H.M. and V. Zizler: Separating polynomials on Banach spaces, Quart. J. Math. Oxford (2), 40 (1989), 409-422.

[FWZ] Fabian, M., Whitfield, J.II.M. and V. Zizler: Norms with locally Lipschitzian derivatives, Israel J. Math. 44 (1983), 262-276.

[G1G2J] Gonzales, M., Gonzalo, R., and J.A. Jaramillo: Symmetric polynomials on function spaces, (1996) preprint.

[G2J] Gonzalo, R. and J.^. Jaramillo: Smoothness and estimates of sequences in Banach spaces, Israel J. Math. 89 (1995), 321-341.

[H] Hanner, O.: On the uniform convexity of $L^{p}$ and $l^{p}$, Ark. Math. 3 (3) (1956), 239-244.

[HeRo] Hernandez, F. and B. Rodrigues-Salinas: Remarks on the Orlicz Function Spaces $L^{\varphi}(0, \infty)$, Math. Nachr. 156 (1992), 225-232.

[LT1] Lindenstrauss, J. and L. Tzafriri: Classical Banach Spaces, Vol. I, Sequence Spaces, Springer-Verlag 1977.

[LT2] Lindenstrauss, J. and L. Tzafriri: On Orlicz sequence spaces III, Israel J. Math. 14 (1973), 368-389. 
[M] Maleev, R.P.: Norms of best Smoothness in Orlicz Spaces, Zeitschrift für Analysis und ihre Anwendungen 12 (1993), 123-135.

[MT] Maleev, R.P. and S.L. Troyanski: Smooth Functions in Orlicz Spaces, Contemporary Math. 85 (1989), 336-351. 